\title{
ADMISSION CONTROL FOR THE CUSTOMERS OVER THE VENDOR'S VPN
}

\author{
G. Narendra Kumar ${ }^{1}$ \\ ECE Dept. \\ UVCE, Bangalore \\ gnarenk@yahoo.com \\ P. Venkataram * \\ PET-UNIT,ECE Dept. \\ Indian Institute of Science, Bangalore \\ pallapa@ece.iisc.ernet.in
}

\author{
V.S.N. Kumar \\ ECE Dept. \\ UVCE, Bangalore
}

\begin{abstract}
There is considerable interest for electronic merchants with limited resources but a limitless ambition for profits, providing quality of service to customers in proportion to their buying potential or finds a legal way of maximizing their profits. The on-line customers always demand fast response and secure transactions, at the same time the electronic merchants are relentless in managing their finite resources to maximize their profits.

We propose a Genetic Algorithm(GA) based call admission control(CAC) scheme for all the customers of a vendor on the vendor's VPN. The algorithm prioritizes the customers based on their past purchase history so that the vendors can select the genuine customer to boost their profits.

Customer Behavior Transition Diagram(CBTD) is used to compute the customer based priority of the vendor. Priorities keep changing dynamically as a function of the status of the customer based on the past purchase history at the ecommerce site. The designed method has been tested analytically and simulated in a real time Internet environment on an e-commerce site consisting of multiple servers distributed on LANs and WANs. This mechanism of admitting clients(customers) on requests among the servers(vendors) at the e-commerce
\end{abstract}


site will improve its revenue at peak times. There is a fervent growth in the business through Internet. Virtual Private Network(VPN) is a must for virtual walk way to make it less vulnerable. Vendor controlled virtual path group(VPG) based VPN is adopted as an appropriate security policy on e-commerce by an architecture dedicated to host and serve the e-commerce site. The results are encouraging in admitting the potential customers and given the optimal service.

Keywords: e-commerce; genetic algorithm; call admission control; prioritized requests; Quality of service; customer behavior transition diagram; virtual private network.

\section{Introduction}

E-commerce services is an important branch of the Internet. With increasing number of Internet users and rapid growth of network technologies, electronic commerce(E-commerce) is perceived as one of the killer applications of the computer and communication technologies. E-commerce can be defined as "buying and selling of information, products and services through Computer networks. The consequence of the popularity is that some e-commerce sites receive more traffic than predicted and congestion occurs due to overload during peak business hours.

A customer who connects to an e-commerce site starts a session that will last some time before the customer has finished the transactions which consists of several requests for varying data and hopefully ends with an order to buy some product. During the whole session the customer expects short response time while requesting new data. If the response time is too long, the customer ends the session and the site will loose its profit from the potential customer. Problems that have been recognized for e-commerce sites are related to site performance and there is a tendency that customers leave and never come back to sites that perform poorly.

The behavior of the customer is also considered during the past visits to the e-commerce site to update the past history and explained using the transition diagram.

Today's VPN solutions overcome the security factor. Using special tunneling protocols and complex encryption procedures, data integrity and privacy is achieved in what seems, for most part like a dedicated point-to-point connection. Using the Internet, companies can connect their remote branch offices, project teams, business partners and e-customers into the main corporate network. Mobile workers and telecommuters can get some connectivity by dialing into the point-of presence(POP)of the load ISP. In this paper we discuss the call admission control for the customers visiting th e-commerce site on priority based on their past purchase history, provide security during transactions by adopting VPG based VPN services for resource allocation and at the same 
time boost the profit of the vendors without compromising QoS.

\section{Call Admission Control to E-Commerce Vendor's Site}

E-commerce sites have limited network resources such as bandwidth and buffers. Hence, it becomes necessary to have some kind of admission control mechanism during congestion occurring at E-commerce site. Further, customers believe that if the site is heavily loaded it is important to receive information about this rather than waiting. If a customer must be rejected, a discount or some kind of 'coupon' should be offered as an incentive to go back to the site. A proper CAC algorithm will ensure prevention of congestion at the sites based on the available resources and prioritized users. Cherkasova and phaal [1] developed a session based admission control, that rejected new customers during congestion.

\section{Introduction to Genetic Algorithms}

Genetic algorithms(GAs), which were introduced by Holland[16], are iterative search techniques based on the the spirit of natural selection. By emulating biological selection and reproduction, GAs can efficiently search through the solution space of complex problems. Genetic algorithms operates on a population of candidate solutions called chromosomes. A chromosome, which is composed of numerous genes, represents encoding of the problem and associates it with a fitness value evaluated by the fitness function. This fitness value determines the goodness and the survival ability of the chromosome. In fact genetic algorithms include; an individual, or a possible solution to the problem; a chromosome, or the parameters that define a solution; a gene, or a parameter; fitness, or how similar a solution is to the desired solution. Gentic algorithm uses the following operators;

Crossover. Crossover [5], selects random genes from parent chromosomes and creates a new offspring.Chromosomes of the two parents are split into two (equal or unequal) halves each. The halves are interchanged and combined to form the child chromosome.

Mutation \& Elitism. After a crossover is performed, the resulting solution might fall into a local optimum - hence some genes of the child chromosome are randomly changed.

However, when creating a new population by crossover \& mutation, the best chromosome might be lost. Hence, Elitism is a method which first copies the best chromosome(s) to the new population. Elitism rapidly increases the performance of the GA, by preventing loss of the best-found solution. 
After the crossover and mutation process is over the offspring is used for replacing some chromosomes in the population with the probability consistent with their fitness values. In other words, GA could construct better and better chromosomes from the best partial genes of past samplings.

In summary, GA is composed of a fitness function, a population of chromosomes and three operators - selection, crossover and mutation. The parameter settings of the operators can be chosen depending on the applications or remain unchanged even when the applications are varied. However, the fitness function and the encoding method are required to be specially designed for each problem.

Genetic algorithm starts initializing the population and evaluating its corresponding fitness values and produces newer generations iteratively. At each generation, a portion of the chromosome is selected according to the survival ability for reproducing offspring. A genetic algorithm operates by the following steps:

1 randomly generating an initial population of solutions, for example numbers, that can be represented in bit-strings,

2 evaluating each individual's fitness via an appropriate fitness function,

3 creating new individuals by mating the current ones by mixing their bit strings together,

4 deleting some or all of the population to make room for new members,

5 repeating all but step (1) for the desired number of generations.

This algorithm is straight forward but involves many variable parameters to be resolved, including:

1 fitness evaluation method, how the mating parents are chosen;

2 mating techniques, how each parent's representative bit string is mixed with others to create new individuals;

3 mutation rate, the probability any bit may change value;

4 population size; and the number of generations.

\section{Proposed Work}

In this paper we present a Call Admission Control mechanism, using Genetic Algorithm, of customers request over the Vendor's Virtual Private Network. 


\section{Rest of the paper organization}

The rest of the paper is organized as follows; Vendor Controlled Virtual Path Group based VPN establishment is described in section 1. Section 2 describes the proposed call admission control for the customers over the vendor's VPN. Simulations and its observations are discussed in section 3. Finally we conclude in section 4 .

\section{Vendor controlled Virtual Private Network for E-Commerce}

The potential customer is selected and resources are allocated by employing the Virtual Path Group(VPG) based VPN which enhances the vendor's capability for Virtual Path(VP) capacity control. It allows transparent signaling and dynamic VP bandwidth allocation within the vendor domain. A vendor can change the VP capacities, within the limits of the VPG capacities without interacting with the provider, Fig.1. Further, the VPG bandwidth can be shared by VPs with different source destination pairs and vendors can independently achieve the optimum balance between the resources needed for VP control to handle the traffic load. In our client-server relation model we have incorporated the Virtual Path Group based VPN as the appropriate security policy dedicated to serve the e-commerce site. VPG is defined as a logical link with in the public network provider's ATM network. A VPG is permanently set up between virtual path(VP) cross connect node and a Vendor Private Network(VDPN) switch that acts as a customer access point for the VPN service. The VPN provider allocates bandwidth to a VPG, which defines the maximum total capacity for all VPs within the VPG.

- Encrypting is made through public key;

- Uses two keys of different type, one public and the other private;

- Owner of the key can encrypt data but can't decrypt;

- Private key is used to decrypt data which is encrypted using public key.

The vendor control contains three controllers: VP admission controller, VPG controller and the VPN controller, all operating on different time-scales to run asynchronously. Assume that one of the VPs experiences a sudden increase in traffic load, the VP admission controller associated with admits all the calls as long as there is sufficient capacity but blocks the calls when overloaded. The VPG controller detects the congestion in that particular VP and attempts to allocate additional bandwidth. If the traffic load is transient the demand for bandwidth drops and the interaction is terminated and if the congestion 
still persists the VPN controller will request additional VPN capacity from the provider.

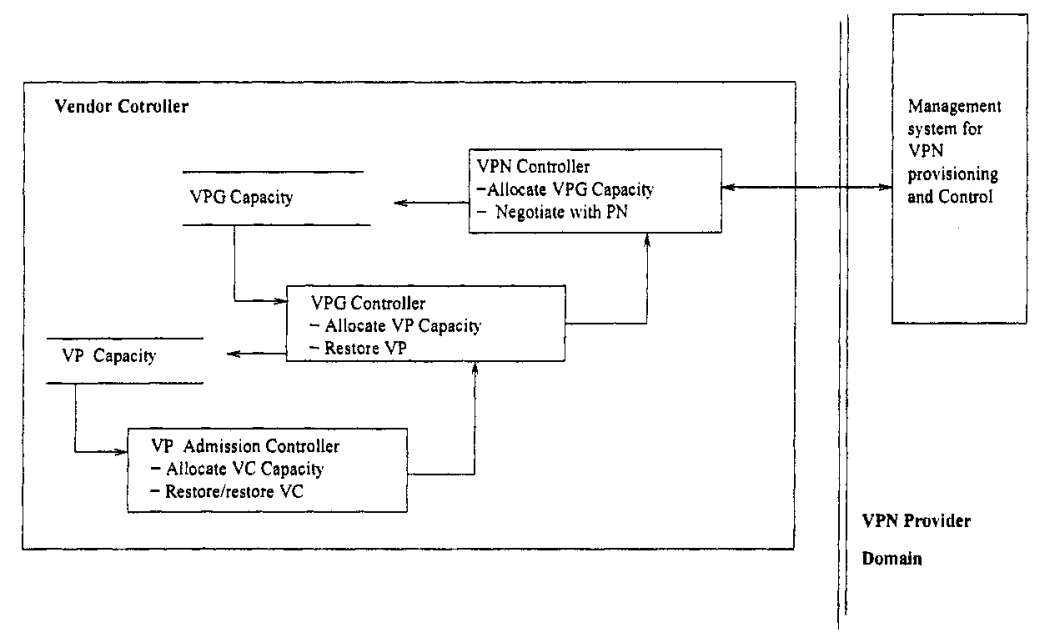

Figure 1. Event Diagram for Vendor control

E-commerce site

Vinual Path

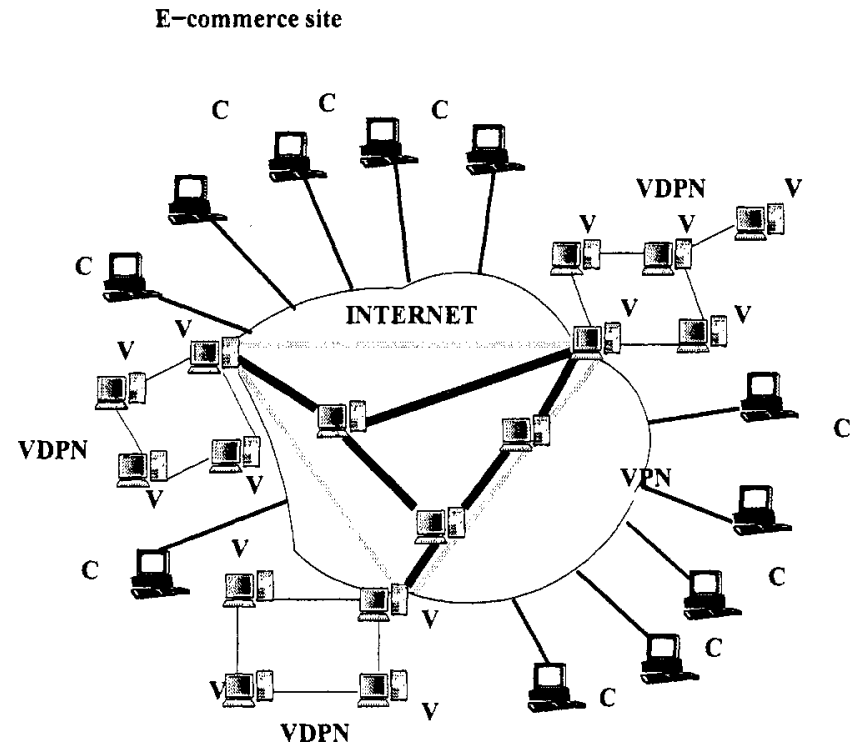

Cajl processing after VPN estabiishment

Figure 2. Call Processing after VPN establishment 
The VP admission controller decides whether a call can be admitted into the VPN based on the VP capacity, its current utilization and the admission control policy, Fig.2. The VP admission controller always ensures that enough capacity is available, such that cell-level QoS can be guaranteed for all calls that are accepted which runs on the timescale of the call arrival and departure rates. Depending on the state of the VPs and the control objectives the VPG controller dynamically changes the amount of bandwidth allocated to associated VPs, enables the customers to exploit variations in utilization among VPs that traverse the same VPG allowing bandwidth between VPs of different source destination pairs to be shared without interacting with the provider. To maintain the QoS, the sum of the VP capacities must be less than or equal to the capacity of the VPG link. The VPN controller dynamically negotiates the bandwidth of the VPG links with the provider, based on traffic statistics and control objectives while observing the customer's QoS requirements.

\section{Proposed Call Admission Control}

In this section we describe a call admission control scheme that may be used in a distributed commercial web site. The control scheme uses the past purchase history of the customers as variables to determine the customer's priority and the vendor's priority to admit the customers. More revenue is generated and at the same time a good quality of service is maintained. The proposed CAC Algorithm admits the clients of the vendor for optimally allocating bandwidth among the E-commerce users. We have designed a Call admission control algorithm using genetic algorithms for e-commerce merchants to increase/maximize the profits obtained from the users/customers by transacting through Internet.

\section{Clients-Vendors relation model}

We have proposed a clients-vendors model in Fig. 3 for implementation of priority based CAC using GA at the router. It represents how the customers are given access to the e-commerce site and allocate the resources available on the vendors secured VPN(virtual private network). The customer requests for entry into the site with a predetermined priority i.e. the customer priority $C_{p}$. The customer priority $C_{p}$ is evaluated based on the past purchase activities at the e-commerce site stored in the database available at the router. Each customer is also given a priority from the vendor's side based on the revenue generated which is termed as vendor's priority $V_{p}$. The client-vendor model discussed aims at finding the genuine customers with good $C_{p}$ and $V_{p}$ to give higher order of preference. 


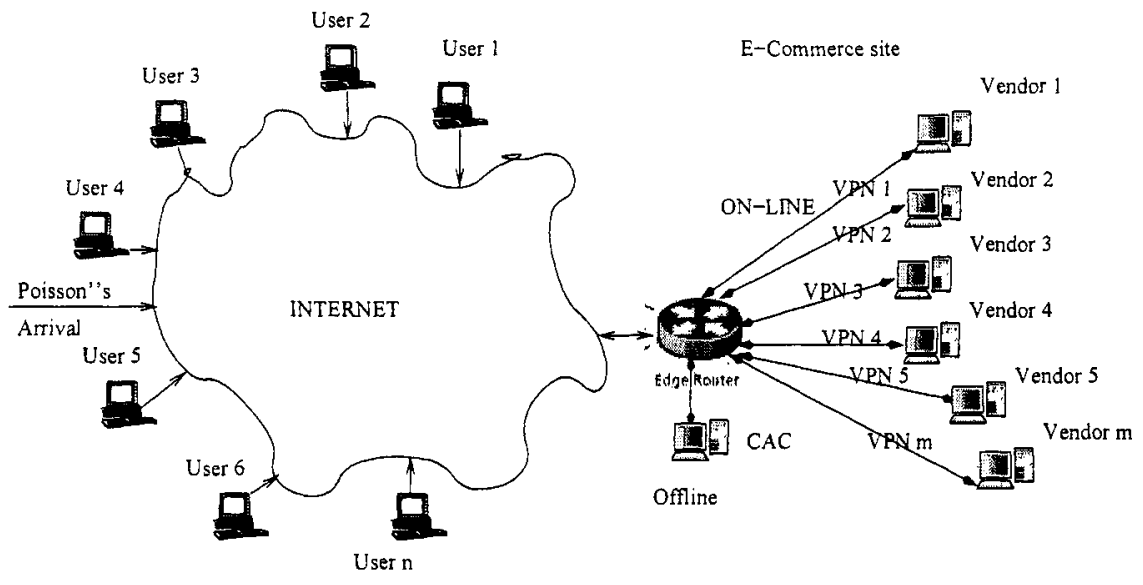

Figure 3. Clients-Vendors Model.

\section{Salient features of the client-vendor relation model}

- Learns the customer behavior and computes the customer buying probability.

- Plans multimedia presentation of the queried information for the customer based on the customer buying behavior.

- Presentation access to the customer is based on resource availability and the customer's past purchase history.

- Admission policy is based on past purchase history of the customers at the e-commerce site.

- Vendors policy is to generate more revenue without compromising with the QoS.

\section{Customer Behavior Transition Diagram}

In a commercial environments, it has been observed that $5 \%$ of the customers stay at home and place orders for necessary goods from the vendor. Evaluation regarding the potential of the customer in terms of profit to the vendor at the e-commerce site is done from the profile of the customer.

We have considered small and heavy buyers as occasional buyers we have built a Customer Behavior Transition Diagram (CBTD)(see Fig.4). The Poisson process is used to model user initiated session arrivals in the Internet to find the transition probabilities in the state diagram. The exit state is reached when 
ever the customer finishes his transactions or when the customer is no more interested at the e-commerce site due to unfriendly navigation, poor contents, a unattractive prices, poor performance and consumes more time to correspond.

The mean time taken by the customer at each state is the 'thinking time' of the potential customers. When a customer has sent a request to the site, he/she waits for the reply. When the reply has arrived, the customer either sends a new request to go to other state or decides to leave the site.

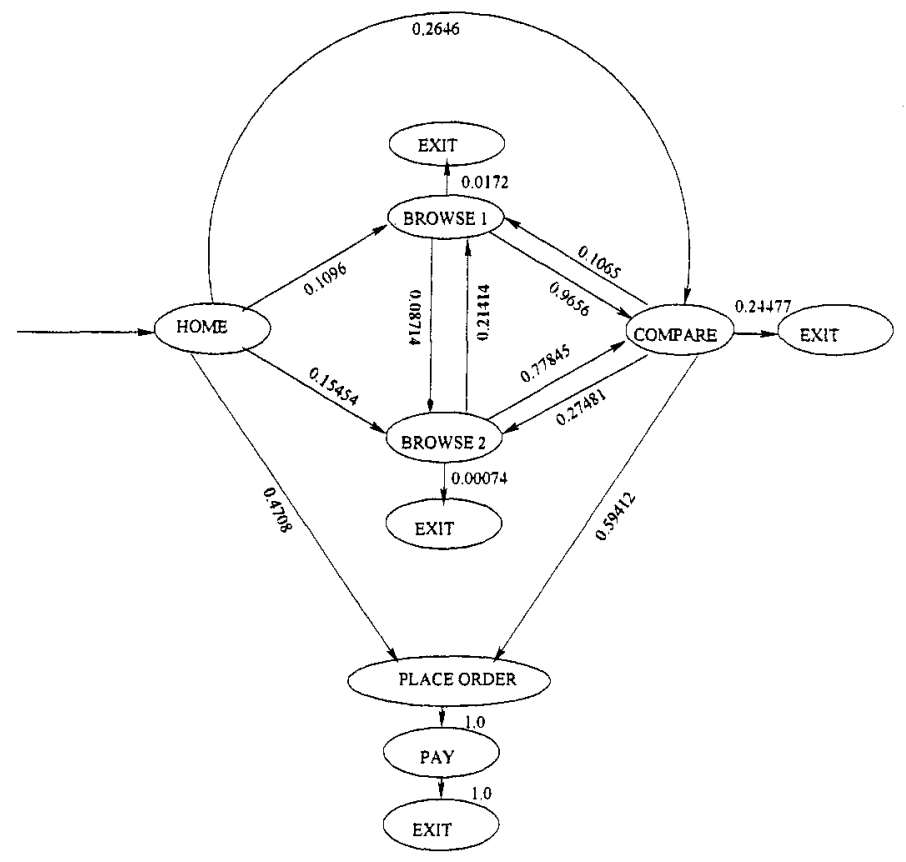

Figure 4. Customer Behavior Transition Diagram

In the CBTD the session length is geometrically distributed. After sending a request of type y the customer will wait for a $S_{y}$ seconds. If the reply does not arrive with in the time the customer becomes impatient and abandons the site by pressing the exit button. The customer visits to the vendor on several occasions and also, at each occasion in which state he/she discontinued the transaction would be taken for the weight-age calculation.

\section{Required Bandwidth Establishment}

For the link the expected $\mathrm{OD}$ (origin-destination) traffic and the link capacity we find the optimal bandwidth assignment which maximizes the total expected throughput and the profit for the vendor. We consider all the customers in the queue at the entry point which is defined as the deviation of assigned capacity 
from the capacity required to give $95 \%$ satisfaction of utility function.

Let ' $C$ ' be the bandwidth available on the particular link ' $i$ ' through which the vendor serves all his ' $n$ ' clients.

We define,

$C_{i}^{\text {link }}$-Available bandwidth of link i, $N_{p}$-Number of unidirectional OD pair $\mathrm{j}$, $U_{j}$-Bandwidth assigned to the OD pair $\mathrm{j}$,

$P_{j}$-Number of predetermined possible paths connecting the OD pair,

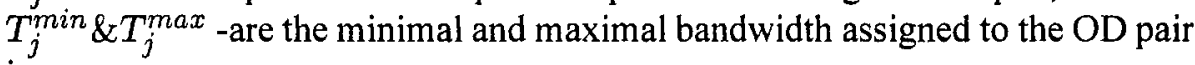
$\mathrm{j}$

$\delta_{j}^{i}$-a indicator variable which takes the value 1 if the OD pair utilizes the link $\mathrm{i}$,

$F_{j}\left(U_{j}\right)$ and $f_{j}(u)$ are the probability function and probability density function for the bandwidth demand.

Then,

$$
\begin{aligned}
& \sum_{j=1}^{N_{p}} \delta_{j}^{i} \times U_{j} \leq C_{i}^{\text {link }} \\
& T_{j}^{\text {min }} \geq U_{j}
\end{aligned}
$$

Analyzing the throughput to be like liquid flow the objective function, $D_{j}\left(U_{j}\right)$

$$
\begin{aligned}
& =\int_{0}^{u_{j}} u f_{j}(u) d u+U_{j} \int_{u_{j}}^{\infty} f_{j}(u) d u \\
& =\int_{0}^{u_{j}} u f_{j}(u) d u+U_{j}\left[1-F_{j}\left(u_{j}\right)\right]
\end{aligned}
$$

we employ $\mathrm{M} / \mathrm{M} / 1$ queuing mechanism for Poisson arrivals,

$$
\begin{aligned}
F_{j}\left(U_{j}\right) & =\frac{\lambda t^{n}}{n !} e^{-\lambda t} \\
F_{j}(u) d u & =\lambda e^{-u \lambda} d u
\end{aligned}
$$

where,

$\lambda$-Mean arrival rate;

$\mathrm{n}$-Number of customers in queue over a interval of time $t$.

\section{- Fitness function evaluation}

Evaluation regarding the potential of the customer in terms of profit to the vendor at the e-commerce site is done from the profile of the customer.

Fitness function

$=\left[\int_{0}^{u_{j}} u f_{j}(u) d u+U_{j}\left[1-F_{j}\left(u_{j}\right)\right]\right]^{*} C_{p_{i}} * V_{p_{j}}{ }^{*} C S_{w_{i j}}$ 


$$
\begin{aligned}
& \text { where, } C_{p}=\frac{(a+2 b+3 c) y z}{10^{5}} \\
& C S_{w_{i j}}=\frac{\Psi}{\tau^{2}}
\end{aligned}
$$

and

$$
V_{p}=\frac{1}{\sqrt{2 \pi \sigma^{2}}} \int_{0}^{V_{p}^{\prime}} \exp -\frac{1}{2}\left({\frac{V_{p}{ }^{\prime}-\mu}{\sigma}}^{2}\right) d V_{p}^{\prime}
$$

The total weight-age of the $i^{\text {th }}$ customer with the $j^{\text {th }}$ vendor is $C S_{w_{i j}}$ with $\Psi$ is the total time spent at the site and $\tau$ is the sum of time allocated for each state transition.

Now a call admission control program is run off-line using GA which gives a list of the priorities of all the customers from the database during congestion of customers at the E-commerce site. The list is compared with all the on-line users and gives access to the customers with the greatest priority. As more customers come on-line the $\mathrm{CAC}$ will check the priority and grant/deny access accordingly. After the customer is granted access the vendor controller decides the dynamic allocation of bandwidth and maintain QoS.

CAC Algorithm. The proposed call admission control algorithm works on taking the several customer's requests to one or several. It is based on many to one and/or to many relationships among the customers and vendors.

\section{Admission policy}

We have adopted following policy in admitting the customers based on the past purchase history with the vendors.

- Customers should be prioritized according to their previous buying patterns and likely wood of buying.

- Infrastructure resources should be assigned to customers according to their priorities.

- CAC is implemented off-line at the router depending on the past history of the customer stored in the database at the vendor.

- History of the customer is available at $C A C$ from all the vendors through user $\log$ file whenever a customer logs on during congestion.

- Every time a customer logs in, a new log file containing the details of number of customers on line and their respective histories from all the vendors is created in a log file. 
- Log file is sent to CAC which seeks a new log file every time it is updated.

- CAC utilizes the history to create priorities to admit customers on priority.

- Every time the CAC receives a updated log file it creates a new priority list.

Customer request for entry into e-commerce site. The customers priority with the e-commerce site, i.e., $C p$ is evaluated. The vendors priority for the customers from the vendors, i.e., $V p$ is evaluated. The GA program is run based on the fitness function to find the priority of the customer seeking access to the e-commerce site. The outcome of the GA program evaluates customer for the allocation of the resources and gives admission.

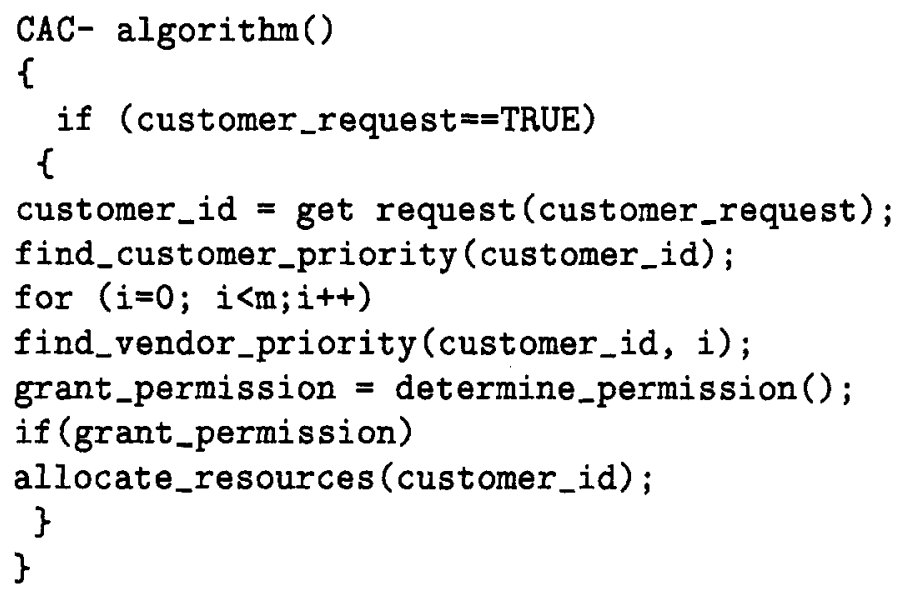

\section{Simulation}

We have carried out the simulation of the customer behavior for customer priority for the e-commerce services on wired and wireless networks resulting in the best solution for maximum profit.

In the simulation we have considered several products and their information at several vendors. We have also considered a case of one product distributed among many vendors based on the information level (surface level, brief note, detailed level, etc). We have tested for several customers needing information and buying the product/s from the vendor/s.

We have simulated this environment at the router and the server of a vendor as a OD pair having $2 \mathrm{MB}$ maximum bandwidth capacity. The mating technique used for all trials was uniform crossover with probability retained at $50 \%$ since there is a balance maintained between exploration and exploitation 
of the population upto 600 generations with population converging after approximately 60 generations and the results were not taken beyond this point. Mutation rate is also important for the mating process which is investigated between $0.2 \%$ and $30.0 \%$ and good results were obtained. This way a list of the fittest strings is obtained and then the CAC checks the on-line customers against this list if the resources are oversubscribed.
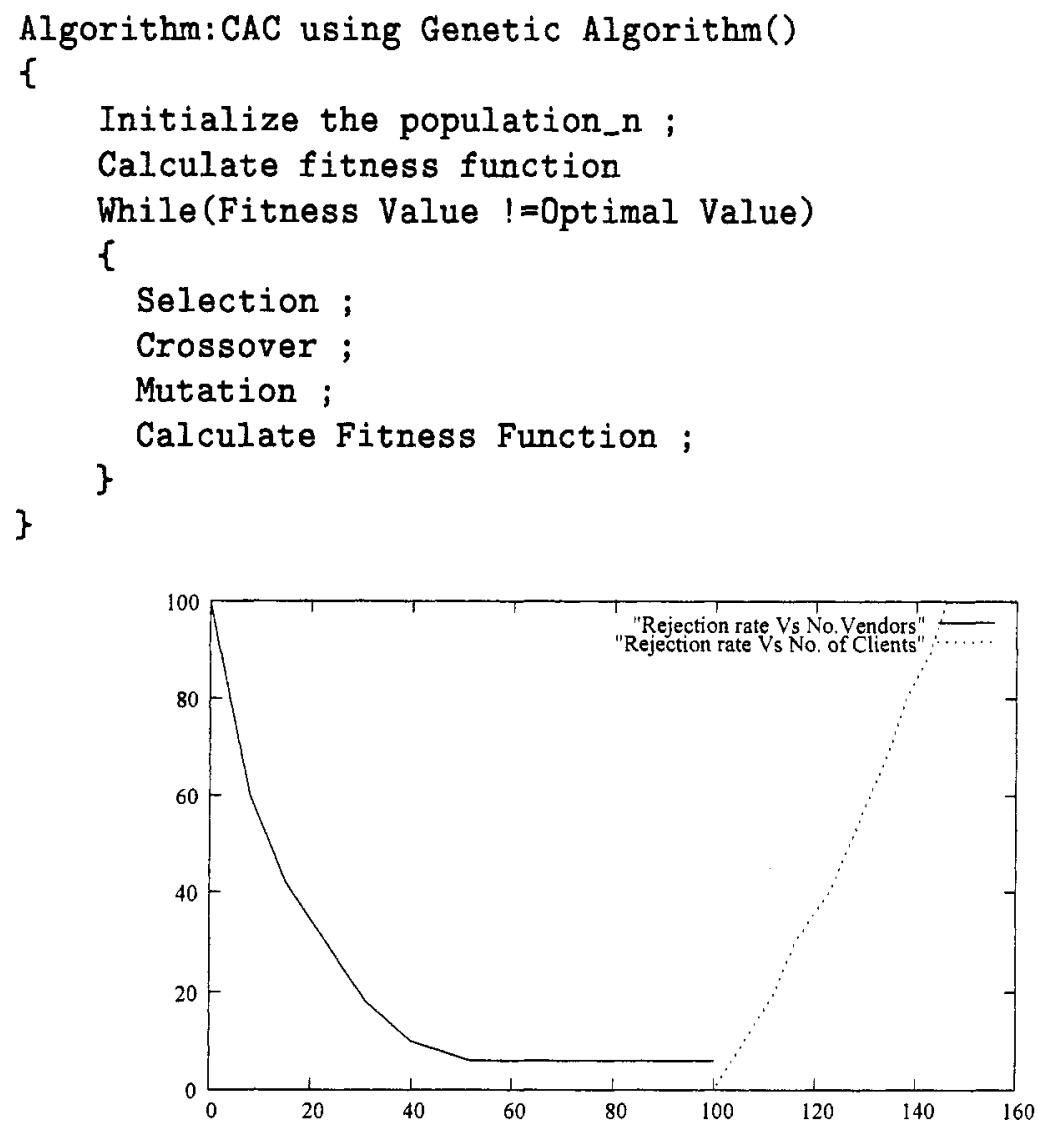

Figure 5. Rejection rate Vs No. of Clients and No. of Vendors

\section{Results}

The results are encouraging in selecting a genuine customer who are the potential customers and given optimal service. In the graph the initial convergence of GA is much faster but at the later stages saturates when run for more than 60 generations. The trend indicates that high mutation rates will approach an optimal value quickly and lower rate will nearly approach the optimal value when the fitness reaches a certain value. 


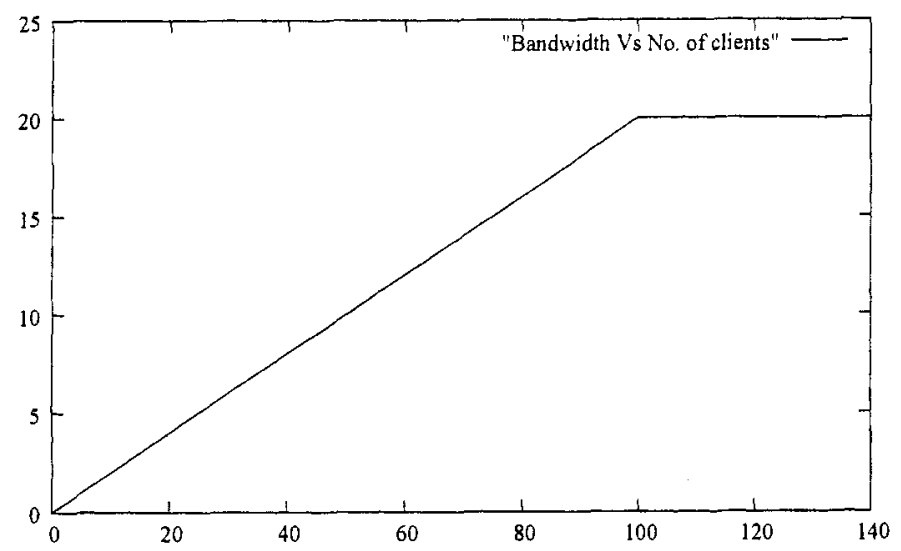

Figure 6. Bandwidth allocation Vs No. of Clients

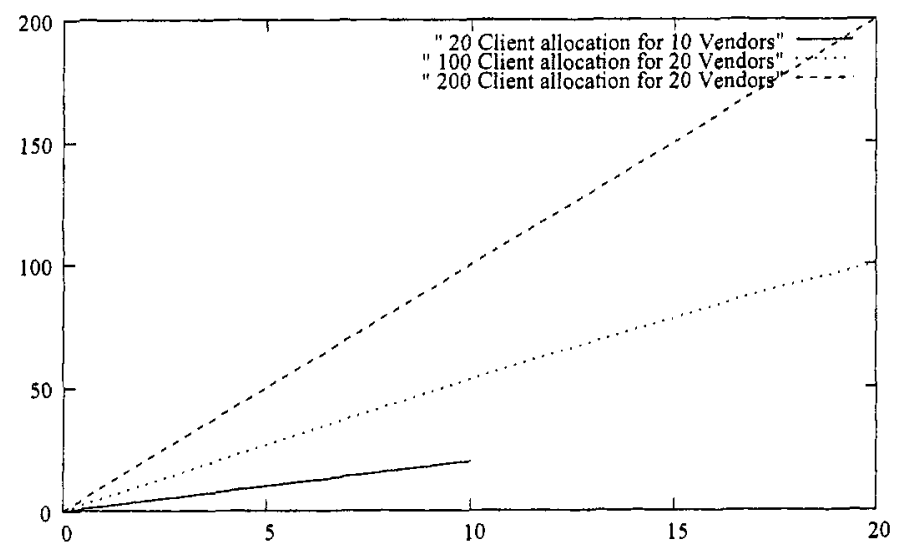

Figure 7. Client allocation Vs No. of Vendors

\section{Conclusion}

The results show that GA is suitable as the complexity of the database at the E-commerce site increases. Working on real time analysis would also lead to better results. The use of combination of the past purchase history of the customer and the behavior of the customer would avoid frustrating potential customers who have been very good buyers there by increasing the revenue throughput of the vendor at the e-commerce site. Further, the VPG based VPN enhances and effectively maximizes the profits of electronic merchants given the physical limits of their infrastructure resources. 


\section{References}

1 L. Cherkasova and P. Phaal, "Predictive admission control strategy for overload commercial web server", Pro. of 8th International Symposium on Modeling Analysis and Simulation of Computer and Telecommunication Systems, 2000, pp 500-507.

2 Davi. E. Goldberg, "Genetic Algorithms in Search, Optimization \& Machine-Learning", Addison Wesley, 1989.

ivalent Capacity and its Applications to Bandwidth Allocation in High Speed Networks", IEEE, JSAC, Vol 9, No.7, Sept 1991.

3 Zhu. L, Wainwright. R. L and Schoenefeld. D. A, "A Genetic Algorithm for the Point to Multipoint Routing Problem with Varying Number of Requests". Proceedings IEEE Intl Conference on EC (ICEC 98), USA, 1998.

4 G.Narendra Kumar, P. Venkataram and V.S.N. Kumar, "A Call Admission Control For E-Commerce Users By Using GA's ",Pro. of International Conference, CIRAS'2003, Singapore, Dec 2003.

5 Stallings, W. "Network and Internet Security: Principals and Practice. "Prentice Hall, 1995.

6 Wasim E. "E-Commerce Systems Architecture and Applications." Artech House, 2000.

7 M.C.Chan, A.A.Lazer and R.Stadler, "Customer Management and Control of Broadband VPN Services", Centre for Telecommunications Research, New York. 